\title{
Breast Imaging Experiences at Academic Medical Centers during COVID-19: Restructuring, Reopening, and Preparing for Possible Resurgence
}

\author{
Stefanie Woodard1, Brian Stahl'2, Elizabeth Allen', Kernesha Weatherly', Angela Choe ${ }^{3}$, \\ Tiffany L. Chan ${ }^{4}$, Chelsea Pyle ${ }^{5}$, Jeong Mi Park ${ }^{1}$ \\ ${ }^{1}$ The University of Alabama at Birmingham, Birmingham, AL, USA \\ ${ }^{2}$ School of Medicine, The University of Alabama at Birmingham, Birmingham, AL, USA \\ ${ }^{3}$ Penn State Health Milton S. Hershey Medical Center, Hershey, PA, USA \\ ${ }^{4}$ Ronald Reagan UCLA Medical Center, Los Angeles, CA, USA \\ ${ }^{5}$ Oregon Health \& Science University, Portland, OR, USA \\ Email: swoodard@uabmc.edu, stahlbp@uab.edu, emolony@uabmc.edu,kweatherly@uabmc.edu, \\ achoe@pennstatehealth.psu.edu,pylec@ohsu.edu,jmpark@uabmc.edu
}

How to cite this paper: Woodard, S., Stahl, B., Allen, E., Weatherly, K., Choe, A., Chan, T.L., Pyle, C. and Park, J.M. (2020) Breast Imaging Experiences at Academic Medical Centers during COVID-19: Restructuring, Reopening, and Preparing for Possible Resurgence. Open Journal of Radiology, 10, 115-126.

https://doi.org/10.4236/ojrad.2020.103012

Received: June 11, 2020

Accepted: July 6, 2020

Published: July 9, 2020

Copyright $\odot 2020$ by author(s) and Scientific Research Publishing Inc. This work is licensed under the Creative Commons Attribution International License (CC BY 4.0).

http://creativecommons.org/licenses/by/4.0/

\begin{abstract}
Severe acute respiratory syndrome coronavirus 2 has thoroughly reshaped the medical landscape. Much has been written and discussed of the adaptations required by this pandemic, particularly in the realm of elective medical care. While some areas of the country have, in recent weeks, seen a plateau or even a decrease in coronavirus 2019 case-burden, others remain face-to-face with significant ongoing morbidity and mortality. Furthermore, given eased restrictions across numerous states and municipalities, a widespread resurgence of the disease is not precluded. To that end, we have attempted to summarize experiences and best practices in the handling of breast imaging against the backdrop of the novel coronavirus, and we consider future directions.
\end{abstract}

\section{Keywords}

Breast, Coronavirus 2019, Scheduling, Resurgence, Planning, Reopening

\section{Introduction}

As the world has changed with the onset of coronavirus 2019 (COVID-19), healthcare has undergone significant modifications to protect patients as well as staff. International organizations have deliberated extensively to discuss the im- 
pact of the pandemic on the medical community. In the United States, major societies such as the Centers for Disease Control and Prevention (CDC), have also provided specific guidance on navigating a new normal of social interaction and disease prevention.

The radiology community has not been immune to the impact of this pandemic. Breast imaging, specifically, is a subspecialty of radiology involving a significant amount of direct patient interaction. Thus, the motivation for reviewing practices in the breast community was prompted by the desire to provide an overview of experiences, changes, and future directions.

Breast radiology involves a combination of essential and nonessential exams. It is important to appropriately triage patients in order to allow for care given to those with time sensitive issues. This is a summary of experiences with rescheduling and altered staffing in breast imaging amidst an evolving crisis. The contributions to current available literature involve reviewing guidelines, expounding on the major recommendations, discussing experiences, and exploring the outlook of breast imaging in the setting of an anticipated second wave of disease.

In this review, the lead author surveyed breast imaging radiologists at four large, academic institutions to obtain each institution's approach to COVID-19. Interviews with these representatives were used to gather information on each institution's decisions to continue or cease the various functions of breast radiology. The institutions whose approaches were considered in this review are located in Eastern, Western, Southern, and Northern United States. Patient characteristics were diverse across catchment areas of these four institutions, which together serve hundreds of thousands of patients annually. We also examined the recommendations of leading radiologic organizations: Society of Breast Imaging (SBI), American College of Radiology (ACR), and American Society of Breast Surgeons (ASBrS). We will discuss both these recommendations and the real-world approaches of the included academic institutions in the setting of a possible second outbreak, which has been suggested in Coronavirus briefings April 22, 2020 by CDC director Robert Redfield [1]. We will also discuss the impact of both closing and reopening, recognizing that recommendations and guidelines continue to evolve as the situation progresses.

\section{Experiences in Restructuring a Breast Radiology Section by Scheduling, Staffing, and Continued Reassessment}

\subsection{Multidisciplinary Approach}

In breast health, as in all of medicine, scheduling and rescheduling are dynamic and multidisciplinary activities. Before any action takes place, multiple discussions are needed between breast radiology, breast health providers, surgical oncology, medical oncology, and radiation oncology. Each program represented in this review expressed the importance of patient coordination with clinical, surgical, and oncologic visits, especially in times of crisis. The institutions included herein all describe holding multidisciplinary conferences via video, which has 
been essential to coordinate patient management. Additionally, radiologists have remained available remotely to take consults, attend remote meetings, and field questions. With this team approach, no major conflicts have been encountered in our cohort.

\subsection{Patient and Exam Scheduling}

\subsubsection{Screening}

Some delay in screening has been required to adhere to best social distancing practices and prevent COVID-19-related morbidity. This has not only been the practice of multiple breast imaging centers, but was encouraged by national organizations including the SBI [2], ACR [3], and ASBrS [3] [4]. Their recommendations are to balance social distancing and the cessation of nonemergent exams with the need to expeditiously manage breast cancer. To this end, the recommendation is to delay screening for several weeks up to a few months [2] [3] [4]. Continuous reassessment of this dynamic situation is suggested to ensure appropriate management of these asymptomatic patients. This screening discussion includes mammography, magnetic resonance imaging (MRI), and ultrasound.

Mammography: The most widely-adopted approach for screening mammography has been to cancel/reschedule all screening mammograms. This approach is supported by the aforementioned organizations. Screening mammograms in our surveyed group were postponed for two to four months. In the setting of a resurgence, screening mammograms should once again be halted with a similar approach of delay/reschedule.

MRI: A primary screening modality for high-risk patients is breast MRI. While the joint ASBrS and ACR statement does recommend postponing screening MRI [3], we have noted a heterogeneous response. Observations made by Moy, et al. in Journal of Breast Imaging [5] and our own authors' experiences indicate that screening via breast MRI has continued at institutions in the Eastern/Western United States. Approaches ranged from complete cancelation to continued normal screening. Reasons for continuing MRI exams may include the elevated patient population lifetime risk for breast cancer, difficulty in MRI scheduling, and potential revenue loss. Given the relatively small patient population needing MRI and the overall greater lifetime risk of patients involved, one approach has been to individually evaluate each MRI indication.

Ultrasound: Another screening modality is automated whole breast ultrasound (ABUS), which is performed at two of the four institutions surveyed. Per guidelines, these exams were managed the same as screening mammography [3].

\subsubsection{Diagnostic}

Mammography: Diagnostic patients involve a mixture of time-sensitive and deferable exams. Schedules should be evaluated on a case-by-case basis, which has been practiced by many institutions [5]. We believe that successful management involves patient satisfaction, safe and effective patient care, and provider welfare, 
which each surveyed institution believes to have achieved to varying degrees. From our experiences and the guidance of national organizations, we present the following management rationale and considerations for COVID-19 recurrence planning:

Exams rescheduled:

1) Patients with a history of breast cancer presenting for routine imaging after treatment.

a. These are considered "Priority C" in the ASBrS recommendations, which suggest a delay in "services ... for the duration of the COVID-19 pandemic" [4].

2) Patients presenting with isolated breast pain.

a. Breast pain alone has been found to carry no significantly higher risk of malignancy than that seen in screening mammography [6].

3) Patients scheduled with a prior exam of Breast Imaging Reporting and Data System (BI-RADS) 3 for standard follow-up.

a. The low likelihood of malignancy of these findings $(<2 \%)$ warrant delay in imaging [7].

It should be noted that our cohort varied significantly in practice mainly based on their geographic setting, patient volume, and institutional/state guidance provided.

Exams not rescheduled:

1) Patients with active untreated breast cancer in need of further evaluation or monitoring response to therapy.

a. This has been the recommendation from the ASBrS if based on a "Priority B" assessment [4].

2) Screening callbacks (patients recalled from screening mammography).

a. Some continued to evaluate callbacks while others handled these exams on a case-by-case basis. This variability may depend on institution volume, staff available to call/reschedule, or concern for medical-legal implications. Some cases may be considered safe to delay work-up. For example, grouped coarse heterogeneous microcalcifications on a screening mammography exam in a breast with almost entirely fatty tissue may indicate only low-grade ductal carcinoma in situ (DCIS). However, filtering through a large pool of callback patients to select this small subset of exams may not be efficient for some institutions.

3) New palpable lumps*.

4) Skin changes*

5) Nipple discharge*

6) Any new symptoms potentially suggesting malignancy*.

*As suggested by the SBI, "clinically concerning symptoms", may warrant evaluation in a timely fashion [2], and in the appropriate setting, these presentations could be considered by many breast health providers and breast radiologists as worrisome.

Nonetheless, a balance is needed between timely assessment of troubling clin- 
ical findings and consideration for immediate patient safety. Epidemiologic assessment may play a role in this difficult decision. Physicians may defer diagnostic evaluation of patients at high-risk of COVID-19 morbidity if local virus prevalence, either in the hospital or in the community, is great enough to suggest significant risk to the individual. To date, no risk-stratification algorithm exists to correlate individual patient risk for contraction of the virus with the local prevalence of the disease. Undoubtedly, this is a difficult decision for doctors to make, and a great deal of careful judgment is required on the part of the physician.

Ultrasound: As with mammography, ultrasound exams scheduled for pain or follow-up of BI-RADS 3 lesions were rescheduled. Those scheduled for evaluation of new cancers, monitoring disease progression, or new symptoms remained as scheduled. The same considerations described previously should inform the decision for diagnostic ultrasound.

MRI: All breast MRI exams performed for the purposes of evaluating extent of disease, rare diagnostic conundrums, axillary metastasis from unknown origin, concern for anaplastic large B-cell lymphoma, or any work-up for possible malignancy have been performed as originally scheduled at all institutions included in this review. Additionally, some have continued MRI exams for BI-RADS 3 findings, post-biopsy evaluation, and follow-up after cancelled biopsies (as is standard for non-visualization of lesions on the day of MRI-biopsy). At those institutions that have continued these exams, the rationale has been that these patients are typically of elevated lifetime risk. Others have considered these exams non-urgent, rescheduling just as with mammography and ultrasound [5]. It should be noted that there is less robust data regarding follow-up of BI-RADS 3 on MRI than there is for mammography [8].

\section{Staffing}

\subsection{Radiologist Scheduling}

Regardless of the specific number of breast radiologists on staff, the trend has been to significantly decrease the number of radiologists physically present. The institutions surveyed here reported an approximately $60 \%$ reduction of on-site faculty present.

Weekend call schedules vary among breast radiologists with some not participating in call, others taking regular call, and many participating in "breast-only" call. Breast-only call may or may not be needed given a low workload. General call may allow for some to participate in sections where a different need presents. Discussion amongst each radiology practice should determine who should/should not be offered the opportunity to be clinically assigned. Radiologists who have been identified as "at risk" by the Centers for Disease Control and Prevention (CDC) may be those with HIV, asthma, higher risk for severe illness, and older adults. Older adults have been described by the CDC as those over age 65 [9].

While remote reading and at-home workstations are ideal for limiting radiol- 
ogist exposure, breast radiologists have roles beyond image interpretation that may preclude this option. Procedures require the radiologist to be on-site. Additionally, many breast radiologists strongly favor in-house ultrasound evaluation and the ability to personally scan patients at the time of diagnostic work-up. Another limiting factor is the FDA regulation for mammography-specific monitors. These regulations require the use of five-megapixel display screens and continuous testing by a qualified medical physicist. Given these factors, some institutions found remote reading for breast imaging to be a poor use of resources. However, Moy, et al. note that other practices have found home workstations to be a suitable alternative in the setting of appropriate supplies and availability [5]. For institutions not equip with home workstations, rapid workstation deployment has also been shown effective as reported recently ahead of print by Tridandipani, Holl, and Canon [10]. In addition, both the report from SBI and our survey have found that most breast radiologists were able to access the electronic medical records remotely, allowing for follow-up on pathology results, preparing conference presentations, and continuing research projects.

\subsection{Resident and Fellow Scheduling}

We found that most fellows were assigned fewer days and less time on clinical service, which is consistent with what was recently reported by Moy, et al. [5]. Fellows were selectively assigned based on their need to gain experience (i.e. Breast MRI and MRI-guided biopsies, which are lower volume) and may also participate/lead conferences through video meetings.

Residents are often not essential for the functioning of breast sections in radiology. While most institutions continue to schedule residents for call shifts, their presence in the outpatient setting of breast imaging may be considered unnecessary exposure. Some sites have eliminated residents from the breast rotation, whereas others have reduced their days and/or hours of service. During time that residents are not clinically assigned, they may prepare and participate in multidisciplinary conferences and journal clubs. This is a requirement at some institutions. One institution has a pre-built mammography screening file with a dedicated room for resident review. Other sources for resident education include, RadPrimer, RadExam, and online educational activities provided by multiple national radiology organizations. Resident didactics may be continued by online video conference. The radiology review committee of the ACGME has developed stipulations to allow for completing graduation requirements during this time, lessening the burden on resident scheduling. As sections return to normal workflow, residents have been scheduled to alternate between academic and clinical work. Isolated workstations have been preferred for residents and will likely persist given the implementation of social distancing expected over fall and winter of 2020 .

The role of students, including medical, graduate, and undergraduate, has posed a challenge. Early guidance from the Association of American Medical Colleges (AAMC) was to remove all students from clinical involvement. More 
recent communications have given more nuanced recommendations. Currently the AAMC recommends that local severity guide decision making [11]. Hospitals in hard-hit locales or with PPE shortages should continue to exclude students from patient care activities. Hospitals with adequate PPE stores and located in areas with a less severe outbreak may exercise some flexibility in student scheduling. However, the AAMC continues to recommend that student activities be limited only to those that are required for graduation/progression in medical education [11]. Given this recommendation and given that radiology rotations are not widely required for medical students, the inclusion of students in radiology sections was not recommended. Students may be returning in the winter of 2020; however, this will be site specific given the varying viral case numbers at different institutions.

Multiple institutions have found that medical students, with newfound free time, are seeking additional academic endeavors. Where possible, students should be given the opportunity to expand their academic horizons and participate in research opportunities remotely. Additionally, Aquifer and other online courses may be utilized for remote teaching of medical students.

\subsection{Technologist Scheduling}

With the cessation of screening, the number of technologists required to staff each facility has significantly decreased. The institutions surveyed report between a $30 \%$ to $50 \%$ reduction in hours worked by technologists, relative to normal operations. The roles of technologists are many, from mammography to ultrasound to assisting in procedures. They also perform innumerable clerical tasks throughout the day. Some facilities may be able to utilize one technologist in multiple roles; however, others necessitate multiple individuals staffing each position. One possibility for scheduling is allowing qualified technologists the option of redeployment in different radiology sections. The adoption of bi-weekly staffing has played a role in some practices, and others have significantly reduced workload to shorten workdays, protecting employees. With a return to normal scheduling, the staffing needs are being adjusted with patient volume and in concordance with breast health clinic workflow.

\subsection{Nurse Practitioner Scheduling}

An advanced care practitioner (ACP), such as a nurse practitioner (NP) or physician's assistant (PA), may aid in many aspects of patient care. These areas include patient follow-up, new patient outreach, and interdisciplinary coordination between breast health clinics and breast radiology. For any new model of staffing, specific roles and responsibilities should be discussed between the ACP, faculty, and administration. Telemedicine appointments have been utilized. Some ACPs may assist in procedures. One site employs both a nurse and medical assistant (MA) and asks them to alternate staffing. Others have limited the ACP's days of service to one to two days per week or assigned the ACP an administrative role. This scheduling model could be reintroduced in the setting of 
a resurgence as many have found telemedicine suitable.

\subsection{Administrative Support}

Dedicated administrative staff often assists in clinical support and coordinating patient services. Typically, assignments include reading room assistance, scheduling, MRI screening call-backs, biopsies, and outside consults. Additionally, liaisons between breast radiologists, breast health clinicians, and breast surgeons are helpful in academic medical centers. Staff is often needed for performing MQSA audits, patient navigation, and patient greeting. The academic institutions surveyed here have reduced their administrative staff's overall hours by up to $60 \%$. Scheduling remains important in the face of numerous cancelled and rescheduled appointments, and some sites have kept at least one scheduler working as usual, remotely when possible.

For continued personalized care, the lead author's institution chose to keep one person physically present as a patient care coordinator. This was felt to be important in forming relationships with patients. This person is responsible for scheduling appointments and biopsies, providing direction, and answering any outstanding questions. Many medical centers have forbidden guests from accompanying patients to appointments, which can be overwhelming and frightening for some. Having a personal interaction may provide comfort during these encounters. At some institutions, there may be visitor restrictions for several months to a year. If a recurrent rise in cases occurs, patients may be attending appointments alone for the foreseeable future. This highlights the need to ensure patient emotional support amidst this isolation.

\subsection{Protective Equipment and Infection Reduction Techniques}

Protective equipment has been a controversial topic. All institutions responding to this inquiry confirmed the use of masks for every patient encounter. Most do not have N95 masks and surgical masks are more commonly used. Gowns, gloves, and facemasks have been endorsed during biopsy procedures. If N95 masks are used, ultraviolet germicidal irradiation (UVGI) may be utilized to reprocess them until they are visibly soiled, damaged, or fail to maintain their seal. Most institutions now require faculty and staff to don a protective mask before entering any building.

The use of face masks by the general public has significantly expanded since the beginning of the pandemic. In early April, the CDC released guidance urging individuals to wear protective cloth face coverings wherever social distancing is difficult to maintain, particularly in indoor spaces [12]. Most hospitals now require all individuals entering for any reason to wear protective face covering.

COVID-19 testing is not routinely performed for asymptomatic diagnostic patients. Some institutions; however, may test patients who are scheduled for biopsy. Patients are scheduled 72 hours in advanced to allow adequate time for testing. This can be communicated by an ACP and scheduled by a nurse trained 
in the process of COVID-19 testing. If the test comes back positive, administration contacts the ordering physician directly. In these cases, the breast radiologist is involved in discussing management of the patient's breast care.

Rooms, tables, door handles, and beds are routinely cleaned by staff throughout the day. Accelerated and more vigorous protocols have been implemented by different groups, including the department of environmental services. Social distancing is uniformly practiced at all institutions, maintaining a distance of six feet between waiting room patients. Hospital administration, department chairs, and leaders in infectious disease monitor national guidelines and have managed protective efforts.

\section{Continuous Reassessment and Re-Entry}

Re-entry into the workplace is governed by federal, local, and individual hospital administration recommendations. Breast section employees should anticipate guidance regarding a "return to work" from these organizations. There are different techniques institutions have employed to ensure safety of patients and providers. The lead author's institution has enacted a campus reentry course. This involves learning activities to ensure that safety is a priority with different phases of return to normal. Guidelines have been set, continued assessment performed, and improved environmental health stressed. Facemasks are universally recommended in our surveyed cohort. Uniformly at all institutions, at least cloth facemasks are required unless in a private space. Careful hygiene is encouraged with appropriate handwashing, sanitizer use (at least $60 \%$ alcohol), social distancing (at 6 feet minimum between individuals) including no congregating of more than 10 people. Electronic meetings continue to be endorsed across institutions. Organizations have been encouraged to continue to limit personnel, avoid items that are frequently touched, use stairs instead of elevators, and disinfect commonly handled surfaces. Some additional steps that may help with reducing transmission include cleaning individual work spaces, avoid sharing personal computers/workstations, and disinfecting landline telephones. Importantly, staying home when sick is crucial at this juncture. While these policies are not specific to breast radiology, compliance is needed from a teamwork perspective.

\section{Revenue and Future Direction}

The current best practices for breast radiology sections, while necessary to slow the spread of the virus, are not without their own challenges. The need for strict social distancing practices has decreased radiologic volume across all subspecialties. A report published by RSNA in April 2020 notes that inpatient radiology volumes across the country have dropped $50 \%$ relative to their levels before the crisis, and some outpatient volumes have fallen by $70 \%$ or more. Furthermore, mammography volumes plummeted $90 \%$ - 95\%. The institutions included in the present survey can confirm that, anecdotally, these percentages from Forman and Cavallo have been accurate [13]. With this loss in volume comes a conco- 
mitant loss in revenue. Many practices are already feeling this acute decrease. The ability of each group to handle decreased revenue will depend on a multitude of factors. At academic centers, breast radiology is one of many services for whom a majority of revenue is derived from elective cases. Hospitals will have to address the losses of outpatient radiology, medical, and surgical services alike. Federal assistance will be required, which will take the form of both loans and deferrals [13] [14].

While patients and physicians alike are eager to resume previously scheduled appointments, a return to normal operations should not come without careful planning. The CDC and its representatives have continued to urge widespread public use of facemasks, and breast imaging centers should ensure their patients adhere to these guidelines [12]. Anecdotally, the home institution of the lead author noted a profound decrease in healthcare worker disease transmission as soon as universal facemask use became hospital-wide policy. Social distancing will remain critical too, and if practices wish to increase study volume, solutions must be put in place to ensure adequate distance between patients. One approach may be to increase hours of operation, so that fewer patients are waiting to be seen at any given time.

Furthermore, a resurgence of viral cases (sometimes described as a "second wave") is not precluded by present plateaus and decreases in incidence. Should such a situation arise either locally or nationally, rapid downscaling may be necessary. Schedulers and support staff should establish expectations for such a contingency as they contact patients to return for delayed and cancelled exams. Additionally, given that any return to normalcy will remain tenuous until definitive treatment or a vaccine is developed, breast sections should reschedule exams in the order of what could least afford to be delayed, as outlined above.

\section{Conclusions}

In summary, taking assertive and quick action to reduce both patient throughput and unnecessary staffing is essential to the safety of the community during a time of a viral pandemic such as COVID-19. The key points to consider include staffing alterations, patient scheduling and rescheduling, and restructuring trainee curricula.

- Staffing changes should include all levels of personnel, including radiologists, fellows and residents, medical and undergraduate students, ACPs, technologists, administrative support staff, and patient care providers.

- Patients should be triaged appropriately and rescheduled with anticipation of resurgence.

- The institutions participating in this discussion have multiple levels of trainees, requiring implementation of appropriate educational venues.

These models of rescheduling and limited staffing apply mainly to academic medical centers with a large number of referrals; however, most of the principles listed above can be applied to non-academic practices as well. Maintaining prompt evaluation of patients with oncologic and urgent breast issues, limiting 
exposure for those with very low risk, and protecting faculty, staff, and learners are imperative to a breast imaging section in these times. We hope that this summary along with previously published responses may provide guidance for during this current pandemic, the recovery process, and the anticipation of a possible resurgence.

\section{Conflicts of Interest}

The authors declare no conflicts of interest regarding the publication of this paper.

\section{References}

[1] Washington Post. May 21, 2020.

https://www.washingtonpost.com/health/2020/04/21/coronavirus-secondwave-cdcd irector

[2] Society of Breast Imaging. Statement on Breast Imaging during the COVID-19 Pandemic.

https://www.sbi-online.org/Portals/0/Position\%20Statements/2020/society-of-breas t-imaging-statement-on-breast-imaging-during-COVID19-pandemic.pdf

[3] The American Society of Breast Surgeons and the American College of Radiology. ASBrS and ACR Joint Statement on Breast Screening Exams during the COVID-19 Pandemic. https://www.breastsurgeons.org/news/?id=45

[4] The American Society of Breast Surgeons. Recommendations for Prioritization, Treatment and Triage of Breast Cancer Patients during the COVID-19 Pandemic: Executive Summary.

https://www.breastsurgeons.org/docs/news/The COVID-19 Pandemic Breast Can cer_Consortium Recommendations_EXECUTIVE SUMMARY.pdf

[5] Moy, L., Toth, H.K., Newell, M.S., Plecha, D., Leung, J.W.T. and Harvey, J.A. (2020) Response to COVID-19 in Breast Imaging. Journal of Breast Imaging. https://doi.org/10.1093/jbi/wbaa025

[6] Holbrook, A., Zhang, J., D'amico, K., et al. (2019) The Association of Breast Pain with Malignancy. Journal of Breast Imaging, 1, 177-181. https://doi.org/10.1093/jbi/wbz029

[7] Sickles, E.A., D’Orsi, C.J., Bassett, L.W., et al. (2013) ACR BI-RADS ${ }^{\circledast}$ Mammography. In: ACR BI-RADS Atlas, Breast Imaging Reporting and Data System, American College of Radiology, Reston, 247-249.

[8] Morris, E.A., Comstock, C.E., Lee, C.H., et al. (2013) ACR BI-RADS ${ }^{\circledast}$ Magnetic Resonance Imaging. In: ACR BI-RADS Atlas, Breast Imaging Reporting and Data System, American College of Radiology, Reston, 804-813.

[9] Centers for Disease Control and Prevention. (2020) Coronavirus Disease 2019. https://www.cdc.gov/coronavirus/2019-ncov/need-extra-precautions/people-at-hig her-risk.html

[10] Tridandapani, S., Holl, G. and Canon, C. (2020) Rapid Deployment of Home PACS Workstations to Enable Social Distancing in the Coronavirus Disease (COVID-19) Era. American Journal of Roentgenology.

[11] Association of American Medical Colleges. Guidance on Medical Students' Participation in Direct Patient Contact Activities.

https://www.aamc.org/system/files/2020-04/meded-April-14-Guidance-on-Medical 
-Students-Participation-in-Direct-Patient-Contact-Activities.pdf

[12] Centers for Disease Control and Prevention. Recommendation Regarding the Use of Cloth Face Coverings, Especially in Areas of Significant Community-Based Transmission.

https://www.cdc.gov/coronavirus/2019-ncov/prevent-getting-sick/cloth-face-cover. $\underline{\text { html\#studies }}$

[13] Cavallo, J.J. and Forman, H.P. (2020) The Economic Impact of the COVID-19 Pandemic on Radiology Practices. Radiology, 2020, Article ID: 201495.

https://doi.org/10.1148/radiol.2020201495

[14] American College of Radiology. CARES Act Offers Loans and Tax Relief to Radiology Practices.

https://www.acr.org/Advocacy-and-Economics/Advocacy-News/Advocacy-News-Is sues/In-the-April-4-2020-Issue/CARES-Act-Offers-Loans-and-Tax-Relief-to-Radiol ogy-Practices 